\title{
A geometrical model of random porous structures to adsorption calculations
}

\author{
Jan T. Duda $\cdot$ Janina Milewska-Duda $\cdot$ \\ Mirosław Kwiatkowski · Magda Ziółkowska
}

Received: 30 October 2012 / Accepted: 17 January 2013/Published online: 12 February 2013

(C) The Author(s) 2013. This article is published with open access at Springerlink.com

\begin{abstract}
This paper describes a new approach to identification of random porous structures (e.g. present in cheap natural adsorbents, active carbons). It comes from a clustering based description of adsorption process assuming an exponential distribution of adsorbate stack size (the LBET model), combined with the new consistent mathematical relationships between the pore geometry, adsorption isotherm parameters and physical properties of adsorptive. The newly derived formulae are discussed, and results of their application to analysis of an active carbon structure are shown.
\end{abstract}

Keywords Adsorbents · Micropores · Isotherms · Models · Numerical analysis

\section{Introduction}

In cheap adsorbents, e.g. in active carbons a size and shape of pores is highly diversified, so may be characterized in terms of random porous structures. To get information on such structures parameters adsorption measurements and a mathematical model of the adsorption process may be employed. The most commonly exploited technique is

\section{J. T. Duda}

AGH University of Science and Technology, Faculty of Management, al. A. Mickiewicza 30, 30-059 Kraków, Poland e-mail: jdu@agh.edu.pl

J. Milewska-Duda $\cdot$ M. Kwiatkowski $(\bowtie) \cdot$ M. Ziółkowska AGH University of Science and Technology, Faculty of Energy and Fuels, al. A. Mickiewicza 30, 30-059 Kraków, Poland

e-mail: kwiatkow@agh.edu.pl

J. Milewska-Duda

e-mail: milewska@agh.edu.pl based on the BET equation (Jaroniec and Madey 1988) that makes possible to determine the monolayer capacity (used to calculate the material surface area $S_{\mathrm{BET}}$ ) and adsorption energy parameter $B_{A}$, based on low pressure adsorption data. Also the adsorption potential theory (DR model) is used to gain information on pore volume $V_{D R}$ and average surface energy (Clarkson et al. 1997; Kats and Kutarov 1998). More detailed characterization, i.e. pore size and volume distribution, needs a more precise mathematical description of adsorption systems. The most advanced techniques-e.g. based on Density Functional Theory (Tarazona et al. 1987) — are dedicated mainly for materials of a regular porous structure (see e.g. (Jagiello et al. 2006)), and when applied to natural adsorbents, they yield fitting quality comparable to that obtained with the model discussed in this paper (see Duda et al. 2007).

In our earlier works (Duda and Milewska-Duda 2002; Duda and Milewska-Duda 2005; Duda et al. 2005) we proposed a new model describing clustering-based mechanisms of adsorption in random porous structures (the LBET model), involving parameters dependent on the pore geometry. Such parameters values, gained by fitting empirical adsorption data, allow one to infer (in qualitative terms) the pore geometry properties (see Duda and Milewska-Duda 2005).

In this paper we propose to complete the LBET approach by including new mathematical relationships between the LBET model parameters, pore geometry parameters and physical properties of adsorptives. It enables us to gain quantitative information on the pore structure geometry (invariant to adsorptive properties), which may be useful in quick low-cost examination and prediction of adsorption capacity of cheap adsorbents. The newly derived formulae are discussed, and results of their application to analysis of an active carbon structure are shown. 


\section{Adsorption isotherm equation for random porous structures, involving pore geometry effects}

Adsorption modeling in the presented approach is a generalization of the BET model. The multilayer adsorption is viewed as a clusterization of adsorbate molecules in pores, starting at primary sites on the solid surface and limited by geometrical and energetic restrictions for BET-stacks size. Possible creation of branched clusters in larger (hole-like) pores is taken into account (Duda and Milewska-Duda 2002). To handle geometric properties of random porous structures we assumed that the number of primary sites $m_{A k}$ capable to start a stack-like cluster of $k$ particles (limited to $k$ layers) is expressed by the formula:

$m_{A k}=m_{A} \cdot(1-\alpha) \alpha^{\mathrm{k}-1}$;

where $\alpha$ is a parameter of the porous structure. Based on the above we derived the formula for adsorption capacity $m_{a} / m_{A}$ involving the cluster branching ratio $\beta$, i.e. the average number of sites provided by $(n-1)$-th layer for the $n$-th layer, $n=2, \ldots k$, averaged over all clusters ( $\beta=1$ for narrow pores, $1<\beta<\approx 1.5$ for wider ones). For homogeneous surfaces the isotherm equation has the form (Duda and Milewska-Duda 2002):

$$
\frac{m_{a}}{m_{h A}}=(1-\alpha) \frac{\pi}{B_{A}+\pi}+\alpha\left(1+\frac{\beta \theta}{1-\alpha \beta \theta}\right) \frac{\pi}{B_{A}(1-\theta)^{\beta}+\pi}
$$

$$
\begin{gathered}
\pi \stackrel{\text { def }}{=} \frac{f(P)}{f_{s}\left(V_{s}\right)} \frac{1}{B_{C}} ; \quad B_{\mathrm{C}} \stackrel{\text { def }}{=} \exp \left(\frac{Q_{C}}{R T}\right) ; \\
B_{\mathrm{A}} \stackrel{\text { def }}{=} \exp \left(\frac{Q_{A}-Q_{C}}{R T}\right),
\end{gathered}
$$

$f(P)$ is the fugacity of adsorbate at pressure $P, f_{s}\left(V_{s}\right)$ stands for the adsorbate fugacity in its reference state $\left(V_{s}, T\right), V_{s}-$ adsorbate molar volume in clusters, $Q_{A}$ and $Q_{C}$-adsorption energies at the first $\left(Q_{A}\right)$ and higher layers $\left(Q_{C}\right), \theta$-average coverage ratio of the 2 nd and higher layers:

$\theta=\Pi^{*} \cdot\left(\frac{1+w_{H} \cdot \Pi^{*}}{1+\Pi^{*}}\right)$

where

$w_{H}=\frac{\alpha \cdot\left(1+\alpha-\alpha^{2}\right)}{2-\alpha}$ and $\Pi^{*}=\pi(1-\theta)^{1-\beta}$,

$\Pi^{*}$ denotes a transformed relative fugacity, and $\theta$ is calculated recursively.In order to put realistic constraints on $B_{A}$ and $B_{C}$ the following relationships are employed:

$Q_{A}=U_{s}\left(V_{s}\right)-\xi_{A} \cdot Q_{s C}$

$Q_{C}=\left(1-\xi_{a a}\right) \cdot U_{s}\left(V_{s}\right)-\xi_{C} \cdot Q_{s C}$,

where $U_{s}$ is the cohesion energy of adsorbate, $Q_{s}-$ adhesion energy (calculated with the Berthelot rule), $\xi_{A}$-an effective adsorbate-pore contact surface ratio on primary sites on pores (1st layer adsorption), $\xi_{C}$-the same as $\xi_{A}$ for the 2 nd and higher layers, $\xi_{a a}$-as $\xi_{C}$ for adsorbate-adsorbate contacts in stacks (we use constant $\xi_{a a}=1 / 8$ ).

The model assuming $\xi_{A}$ uniformly distributed over the range from $\xi_{A \max }$ to $\xi_{C}$ was derived too (Duda et al. 2005). It describes porous surfaces at which the primary adsorption sites are created by niches of random depth/size, such that the effective adsorbate-solid contact surface fractions $\xi_{A}$ are random numbers of uniform-like distribution. In this case, by appropriate integration of Eq. (2) we arrived at the following formula:

$$
\begin{aligned}
\frac{m_{a}}{m_{h A}}= & (1-\alpha)\left\{1+\frac{R T}{\left(\xi_{A \max }-\xi_{C}\right) Q_{s C}} \cdot \ln \left(\frac{B_{A}+\pi}{B_{f}+\pi}\right)\right\} \\
& +\alpha\left(1+\frac{\beta \theta}{1-\alpha \beta \theta}\right) \\
& \left\{1+\frac{R T}{\left(\xi_{A \max }-\xi_{C}\right) Q_{s C}} \ln \left(\frac{B_{A}(1-\theta)^{\beta}+\pi}{B_{f}(1-\theta)^{\beta}+\pi}\right)\right\}
\end{aligned}
$$

where $B_{A}$ is calculated with Eq. (3) for $\xi_{A}=\xi_{A \max }$, $B_{f}$-like $B_{A}$ for $\xi_{A}=\xi_{C}$.

The models (2) and (6) involve 5 parameters $\left\{m_{A}, \alpha, \beta, \xi_{A}\right.$, $\left.\xi_{C}\right\}$ for vaporous adsorbate, and additionally $V_{s}$ for a gaseous one. In our papers (Duda and Milewska-Duda 2005; Duda et al. 2005) we have shown that low pressure adsorption data $(\pi<0.3)$ make possible to determine well only $m_{A}$ and $B_{A}$ (i.e. $\left.\xi_{A}\right)$. Having data up to $\pi \approx 0.5$, one may estimate $\alpha / B_{C}$, higher pressure data make possible to distinguish between effects of $\alpha$ and $B_{C}$, and if $m_{a}$ for $\pi>0.8$ are available-one may also well estimate the parameter $\beta$.

In the new approach the porous structure is described with 5 parameters invariant to properties of adsorbate molecules. This invariance should allow us to determine a reliable picture of the material porous structure by simultaneous fitting of a number of apolar small molecule adsorptives at the same (studied) adsorbent.

\section{Geometrical model of random porous structures}

In order to facilitate a translation of the model (1-6) parameters into the pore structure parameters (invariant to the adsorbate molecule size) we elaborated an idealized mathematical description of random porous structures (typical in natural adsorbents). It is based on the following assumptions:

1. Each pore is a hole with rough surface made by random removing of molecular particles from the original material (during adsorbent production). A smoothed shape of the pore is a spherical tube ended with hemispheres (see Fig. 1). The smoothed pore parameters: the length $h$ and diameter $d$ are interconnected as follows:

$$
d(h)=A\left(1-e^{-h / A}\right), A=\frac{1.2}{3-b} \quad[n m],
$$


The relation (7) for $d(h)$ means that the pore diameter $d$ enlarges (by a unit), together with the pore length $h$, with a constant probability $(\propto \mathrm{A})$, depending on the material property parameter $b \in<1,3$ ) ( $b$ may be viewed as an anisotropy factor specific for a material). Thus, the only one parameter $h$ may be used to calculate the pore volume $v_{p}(h)$ and evaluate (underestimate) its surface area $s_{p}(h)$ :

$v_{p}(h)=3.14 \cdot \frac{d^{2}}{4}\left(h-\frac{d}{3}\right), s_{p}(h)=3.14 \cdot d \cdot h$,

2. All pores with the same $b$ and random $h$ are packed randomly in the adsorbent space with no volume overlapping, but with numerous random contacts at their surface (making randomly larger channels and holes of more diversified shape)—see Fig. 2

3. Due to the surface roughness in each pore there are two deeper local niches (at the tube ends) making possible to start two adsorbate clusters, i.e. creating two primary adsorption sites (having the locally largest solid-fluid contact surface and appropriately large $\xi_{A}$-see Eq. 5). Moreover, the pore wall roughness create one continuous path at the pore side wall, enabling a bit better adsorbate-solid contacts than at neighboring sites, thus making possible to keep a stack of adsorbate molecules (i.e. having appropriately large $\xi_{C}$-see Eq. 5). The stack-path length $w$ is random and it may vary from $w_{\text {min }}(h)$ corresponding to the shortest connection of the tube ends (primary sites), up to $w_{\max }(h)$ that is close to a spiral length with a fixed lead $L_{w}$. The values $w_{\min }$ and $w_{\max }$ are calculated as follows:

$$
\begin{aligned}
& w_{\min }(h)=\max \left\{h, h-L_{w}+0.57 \cdot d\right\} \\
& w_{\max }(h)=\max \left\{w_{\min }, 3.14 \cdot d(h-d / 2) / L_{w}-L_{w}\right\},
\end{aligned}
$$

where $L_{w}=0.6 \mathrm{~nm}$ is taken (arbitrarily) a bit larger than the largest diameter of typical small molecule adsorbates ( $L_{w}$ is the maximum pore diameter for $b=1)$. The formulae (9) produce $w_{\min }=w_{\max }=$ $h$ for $h<L_{w}$, (we assumed realistically that small micropores may produce only one stack-path of the length $w=h$ )

4. The stack-path length $w$ is exponentially distributed over the set of all stack-paths and in pores of $h>L_{w}$, i.e. the number $m_{p}(w)$ of paths of length $w$ is:

$m_{p}(w)=m_{0} \ln \left(\alpha_{0}\right) \alpha_{0}^{w} d w$

where $m_{0}$ and $\alpha_{0}$ are the pore structure parameters. It means that in any pore an enlargement of the stackpath length by a unit (during the sorbent production) occurs with the same probability $\alpha_{0}\left(m_{0}\right.$ and $\alpha_{0}$ depend on the material properties and production technology).

5. As the consequence of the assumptions (2) and (3), the pore size distribution $g(h)$, pore volume distribution over the pore size $f_{v}(h)$, pore surface distribution $f_{s}(h)$ and corresponding cumulated distributions $G(h), F_{V}(h)$ and $F_{S}(h)$ have to fulfill the following relationships:

$$
\begin{aligned}
& \int_{x=x_{\min }}^{w} g(h(x)) p(w, x) d x=m_{p}(w), \\
& \quad w_{\max }\left(x_{\min }\right)=w, \quad p(w, x)=\ln \left(\alpha_{0}\right) \frac{\alpha_{0}^{w}}{\alpha_{0}^{w_{\max }}-\alpha_{0}^{w_{\min }}} d w
\end{aligned}
$$

$$
\begin{gathered}
f_{v}(h)=v_{p}(h) g(h), \quad f_{s}(h)=s_{p}(h) g(h), \\
G(h)=\int_{x=h_{B}}^{h} g(h) d h, \quad F_{V}(h)=\int_{x=h_{B}}^{h} f_{v}(h) d h \\
F_{S}(h)=\int_{x=h_{B}}^{h} f_{s}(h) d h
\end{gathered}
$$

where $h_{B}$ is a minimum pore size. In our calculations we assumed that in solid adsorbates the minimum pore
Fig. 1 Pore diameter-pore size relationships (left subfigure), and smoothed shape of a series of pores for $b=2$ (right subfigure)
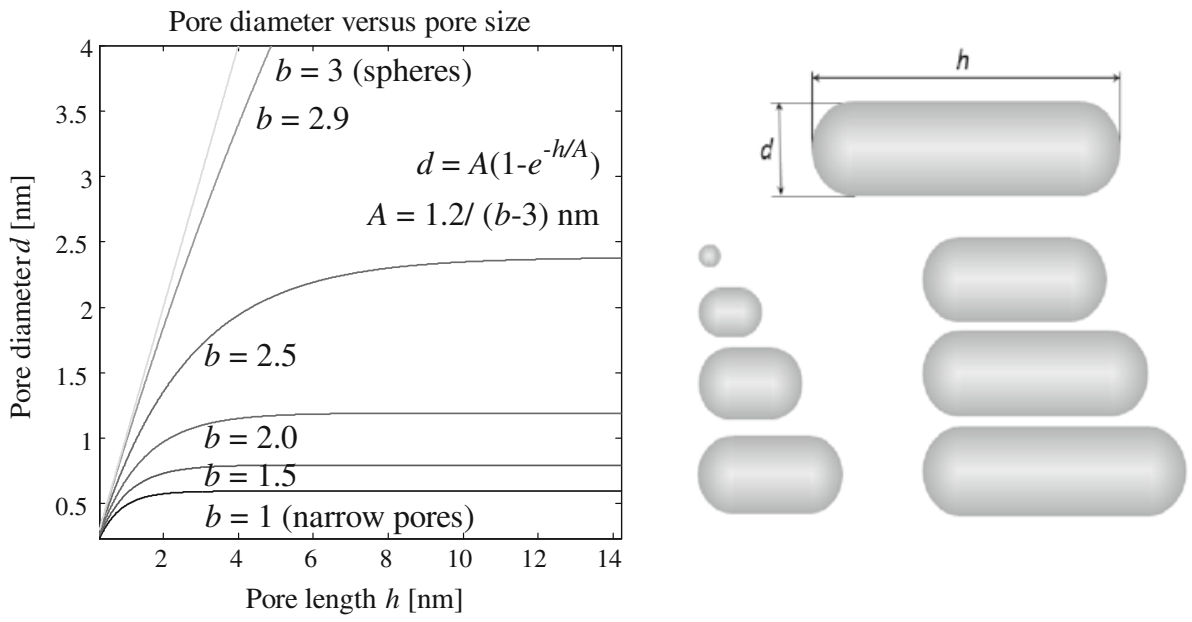
is the cube of volume of aliphatic chain $\mathrm{CH}_{2}$ (it is referred to as the basic volume $\left.v_{B}\right), v_{B}=16 \mathrm{cc} / \mathrm{mol}$, so the corresponding $h_{B}=0.3 \mathrm{~nm}$. The plots for $g(h)$, $f_{v}(h), f_{S}(h), G(h), F_{v}(h)$ and $F_{S}(h)$ calculated numerically for given $\alpha_{0}, m_{0}$ and $b$ are shown in Fig. 3

6. Presence of highly dispersed pores (especially of the smaller ones) reduces an effective adsorbate-solid contact surface, thus decreasing the adsorption energies $Q_{A}$ and $Q_{C}$. This effect may be expressed by a probability $\Phi_{C p}\left(V_{s}\right)$, that a site placed at the geometrical wall of an accessible pore is really a part of this pore wall, i.e. it is placed at the solid matter (not at another pore wall). Having this probability for a given adsorbate $s$, one may correct the parameters $\xi_{A}$ and $\xi_{C}$ in Eq. (5) (expressing an effective adsorbate-pore contact surface area) in the following way:

$\xi_{A}=Z_{A} \Phi_{C p}, \xi_{C}=Z_{C} \Phi_{C p}$

where $Z_{A}$ and $Z_{C}$ stand for the pore structure parameters depending only on the pore surface shape and roughness, at primary sites $\left(Z_{A}\right)$ and at stack-paths $\left(Z_{C}\right)$, respectively. The probability $\Phi_{C p}\left(V_{s}\right)$ may be roughly evaluated, assuming that the solid of the adsorbent is built of basic cubes of $v_{B}$ volume and the surface segments of all pores walls and of solid basic segments are randomly distributed over the adsorbent space. Thus the following simplified relationships were accepted:

$\Phi_{C p}\left(V_{s}\right)=2 \frac{\varphi_{C} \varphi_{a p}\left(V_{s}\right)}{1-\left(\varphi_{C}+\varphi_{i p}\left(V_{s}\right)\right)^{2}}$,

where $\varphi_{C}=\frac{S_{\text {solid }}}{S_{\text {solid }}+S_{\text {pores }}}, \varphi_{\text {ap }}\left(V_{s}\right)=\frac{S_{\text {sacc }}\left(V_{s}\right)}{S_{\text {solid }}+S_{\text {pores }}}, \varphi_{i p}\left(V_{s}\right)=$ $\frac{S_{\text {sin } a c c p}\left(V_{s}\right)}{S_{\text {solid }}+S_{\text {pores }}}$

$\varphi_{C}$ stands for the surface fraction of solid segments, $\varphi_{a p}$, $\varphi_{i p}$ - surface fraction of all pores accessible and inaccessible for the molecules of $V_{s}$ volume (i.e. of $d_{s}$ diameter), $S_{\text {sacc }}, S_{\text {sinacc }}$ denote the surface of accessible and inaccessible pores, $S_{\text {solid }}$-the surface of all solid segments, $S_{\text {pores }}$-the total surface of pores $\left(S_{\text {pores }}=S_{\text {sacc }}+S_{\text {sinacc }}\right)$. The quantities $S_{\text {sacc }}$ and $S_{\text {sin- }}$ acc can be calculated by integration of $f_{S}(h)$ in Eq. (12) over the range $\left\langle h_{B}, h_{\text {sacc }}\right\rangle$ and $\left(h_{\text {sacc }}, \infty\right)$, respectively, where $h_{\text {sacc }}$ is the minimum length of pores accessible for the particle of $d_{s}$ diameter, i.e. $d\left(h_{s a c c}\right)=d_{s}$, and

$h_{s a c c}=-A \cdot \ln \left(1-\frac{d_{s}}{A}\right)$

7. The number $m_{p s}\left(j_{s}\right)$ of stack-paths capable to keep $j_{s}=\operatorname{int}\left(w / d_{s}\right)$ molecules of $d_{s}$ diameter may be calculated by integration of Eq. (10) over $w \in<h_{\text {sacc }}$, $h_{\text {sacc }}+\left(j_{s}+1\right) d_{s}>$ :

$$
\begin{aligned}
m_{p s}\left(j_{s}\right) & =m_{0} \ln \left(\alpha_{0}\right) \int_{\substack{w=h_{s a c c} \\
h_{s a c c}+\left(j_{s}+1\right) d_{s}}}^{\int_{0}^{w} d w} \\
& =m_{0} \alpha_{0}^{h_{a s}}\left(1-\alpha_{0}^{d_{s}}\right)\left(\alpha_{0}^{d_{s}}\right)^{j_{s}-1}
\end{aligned}
$$

We assume that each pore of $h>2 h_{\text {sacc }}$ is capable to keep two adsorbate stacks (starting at its ends) of the same length $k_{s}$ or differing by 1 , i.e. $k_{s}$ and $k_{s}+1$, so that $2 k_{s}=j_{s}$ or $2 k_{s}+1=j_{s}$. Hence, the number $m_{A s}\left(k_{s}\right)$ of stacks limited to $k_{s}$ particles is related to $m_{p s}(j)$ as follows:

$m_{A s}\left(k_{s}\right)=m_{p s}\left(2 k_{s}-1\right)+2 m_{p s}\left(2 k_{s}\right)+m_{p s}\left(2 k_{s}+1\right)$,

It results in the following relationships:

$$
\begin{aligned}
m_{A s}\left(k_{s}\right)= & m_{0} \alpha_{0}^{h_{\text {sacc }}}\left(1+\alpha_{0}^{d_{s}}\right)\left(1-\alpha_{0}^{2 d_{s}}\right) \alpha_{0}^{2 d_{s}\left(k_{s}-1\right)} \\
= & m_{A s}\left(1-\alpha_{s}\right) \alpha_{s}^{k_{s}-1}, \quad \alpha_{s} \stackrel{\text { def }}{=} \alpha_{0}^{2 d_{s}}=\alpha_{b}^{\left(d_{s} / d_{b}\right)}, \\
& m_{A s} \stackrel{\text { def }}{=} m_{0} \alpha_{s}^{\frac{h_{\text {sacc }}}{2 d_{s}}}\left(1+\sqrt{\alpha_{s}}\right)
\end{aligned}
$$

8. An adsorbate molecule placed at a primary site may block the access to other primary sites situated in its vicinity of the surface area proportional to $\left(V_{s}\right)^{2 / 3}$ (it is often referred to as the surface filling effect). Hence, the number $m_{h A s}$ of primary sites, really available for the asorbate $s$, may be calculated as:

$m_{h A s}=m_{A s} \frac{C}{V_{s}^{2 / 3}}$

where $C$ stands for a constant parameter (its value is unimportant for further studies).

That Eq. (18) with $m_{A s}$ replaced by $m_{h A s}$ rewrite the formula (1), when $\alpha_{s}$ denotes the stack size distribution parameter related to the adsorbate of $V_{s}$ volume, and $m_{h A s}$ - the monolayer capacity for this adsorbate. The parameters $m_{A s}, m_{h A s}$ and $\alpha_{s}$ may be calculated by using the native parameters $m_{0}$ and $\alpha_{0}$, or with $m_{A b}$ and $\alpha_{b}$ found before for another ( $b$-th) adsorbate. A picture of pores and porous structures corresponding to the assumptions $1-8$ is shown in Fig. 2.

The parameters $m_{A s}, \alpha_{s}$ and $b$ are interconnected with the adsorption model parameter $\beta_{s}$ (the averaged cluster branching ratio-see Eqs. 2 and 6) due to pore volume constraints. Let $V_{\text {clasts }}$ denote the maximum volume of all clusters possible to be deposed in the material, which is the total volume $V_{\text {psacc }}$ of pores accessible for the adsorbate molecules (of molar volume $V_{s}$ ) reduced by a volume excluded $V_{\text {excls }}$ due to natural discrepancies between adsorbate clusters and pores space shape. Based on the distribution (1) and (18) the following relationship may be derived: 


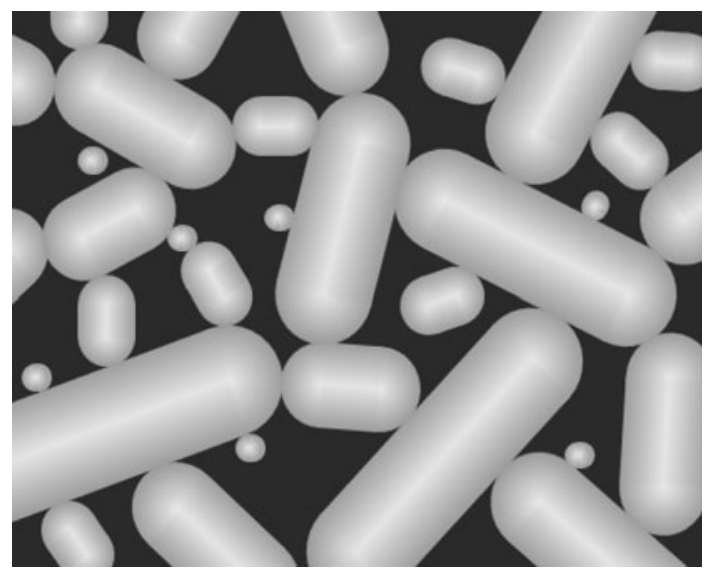

Fig. 2 A schematic picture of random porous structure (pore wall roughness is not shown)

$V_{\text {clasts }}=\frac{m_{A s}\left(1-\alpha_{s}\right)}{\left(1-\alpha_{s} \beta\right)^{2}} V_{s}$,

where $V_{\text {clasts }}=V_{\text {psacc }}-V_{\text {excls }}, V_{\text {psacc }}=F_{V}(\infty)-F_{V}\left(h_{\text {sacc }}\right)$ and $F_{V}(\infty)$ and $F_{V}\left(h_{\text {sacc }}\right)$ are calculated by integration of the pore volume distribution $f_{V}(h)$ in Eq. (12) (like $S_{\text {sacc }}$ in Eq. 14).

Thus, the following relationship is valid:

$\beta_{s}=\frac{1}{\alpha_{s}}\left(1-\sqrt{V_{s} \frac{m_{A s}\left(1-\alpha_{s}\right)}{V_{p s a c c}-V_{\text {exls }}}}\right)$

but $\beta_{s}=1$ should be taken if Eq. (21) produces a value lower than 1 (it may be caused by the model simplifications).

The volume excluded $V_{\text {excls }}$ may be attributed to a fraction of total surface of accessible pores $S_{\text {psacc }}$, where it forms a layer of averaged width equal to $d_{s} / 2$, and this

Fig. 3 Pore size, pore volume and pore surface distributions, $g(h), f_{v}(h), f_{S}(h)$, respectively (upper row subplots) and cumulated pore size, volume and surface distributions, $G(h)$, $F_{v}(h), F_{S}(h)$ respectively (lower row subplots) calculated for $b=2.5$ and $\alpha_{B}=\{0.15,0.30$, $0.45,0.6,0.75,0.9\}$
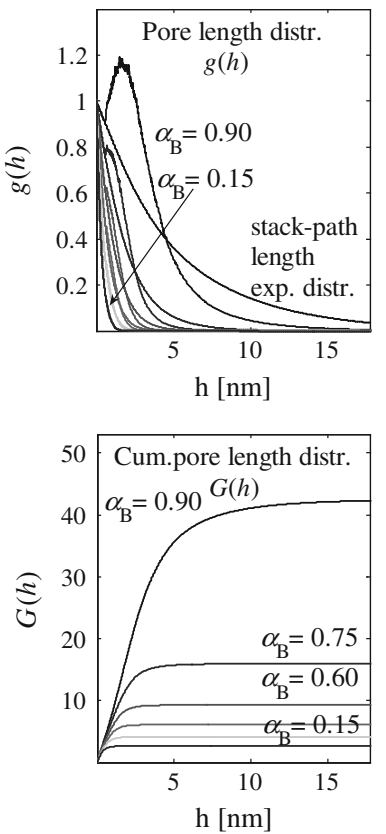
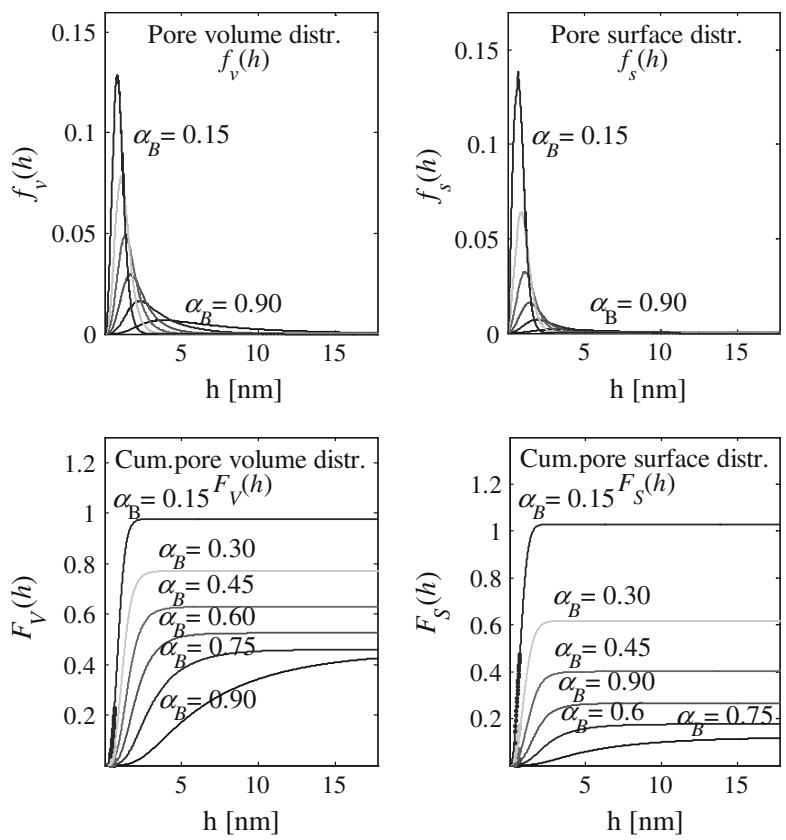

fraction depends on averaged diameter $\left\langle d_{a c c s}\right\rangle$ of accessible pores. Hence, we accepted the following relationship:

$V_{\text {excls }}=\frac{d_{s}}{2} S_{\text {psacc }} \frac{1}{5}\left(1+\frac{d_{s}}{\left\langle d_{\text {accs }}\right\rangle}\right)$

The value for $\left\langle d_{a c c s}\right\rangle$ is roughly evaluated with the following formula (involving Eq. 15):

$$
\begin{aligned}
\left\langle d_{\text {accs }}\right\rangle & \stackrel{\text { def }}{=} \frac{1}{G(\infty)-G\left(h_{\text {sacc }}\right)} \int_{x=h_{\text {sacc }}}^{\infty} g(h) d(h) d h \\
& \approx A \cdot\left(1+\frac{A \cdot \ln \left(\alpha_{0}\right)}{1-A \cdot \ln \left(\alpha_{0}\right)}\left(1-\frac{d_{s}}{A}\right)\right)
\end{aligned}
$$

where $g(h)$ was expressed in the same form as $m_{p}(w)$ in Eq. (10), to simplify calculations.

Properties of the proposed random porous structure description are illustrated in Figs. 3, 4 and 5.

Figure 3 shows the pore size, volume and surface distributions calculated with Eqs. (11) and (12) for $b=2.5$, $\alpha_{B}=\{0.15,0.30,0.45,0.6,0.75,0.9\}$ and pore volume fraction $u_{p}=60 \%$. In the subfigure showing $g(h)$ the pore length distribution $g(h)$ is confronted with the stack-path length distribution $m_{p}(h)$ (dotted lines).

Figure 4 shows other relationships derived in the paper, i.e. $m_{h A}\left(V_{s} / v_{B}\right), \alpha_{s}\left(V_{s} / v_{B}\right)$, and $\Phi_{C p}\left(d_{s} / d_{B}\right)$. It may be seen in the subplot for $m_{h A}\left(V_{s} / v_{B}\right)$ that for high $\alpha_{B}$ the relationships (18) and (19) produce $m_{h A}$ close to the surface filling curve (upper bold dotted line), the curves $m_{h A}\left(V_{s} / v_{B}\right)$ approach to the volume filling curve with decreasing $\alpha_{B}$ (see the lower bold dotted line) and they become lower for $\alpha_{B}<0.5$. The lower left subplot illustrates a relation between $V_{\text {sum }}=F_{V}(\infty)$ and $S_{\text {sum }}=F_{S}(\infty)$, and $V_{\text {exp }}$ and $S_{\text {exp }}$, corresponding to the exponential distribution of pore length $h$ (see Eq. 10). The 
Fig. 4 Transformation formulae for $m_{h A s}\left(V_{s} / v_{B}\right)$ and $\alpha_{S}\left(V_{s} / v_{B}\right)$ (upper row subplots), $V_{\text {sum }} / V_{\text {exp }}, S_{\text {sum }} / S_{\text {exp }}$ and $\Phi_{C p}\left(d_{s} / d_{B}\right)$ (lower row subplots) calculated for different $\alpha_{B}=\alpha_{0}^{d B}$ and $b=2.5$
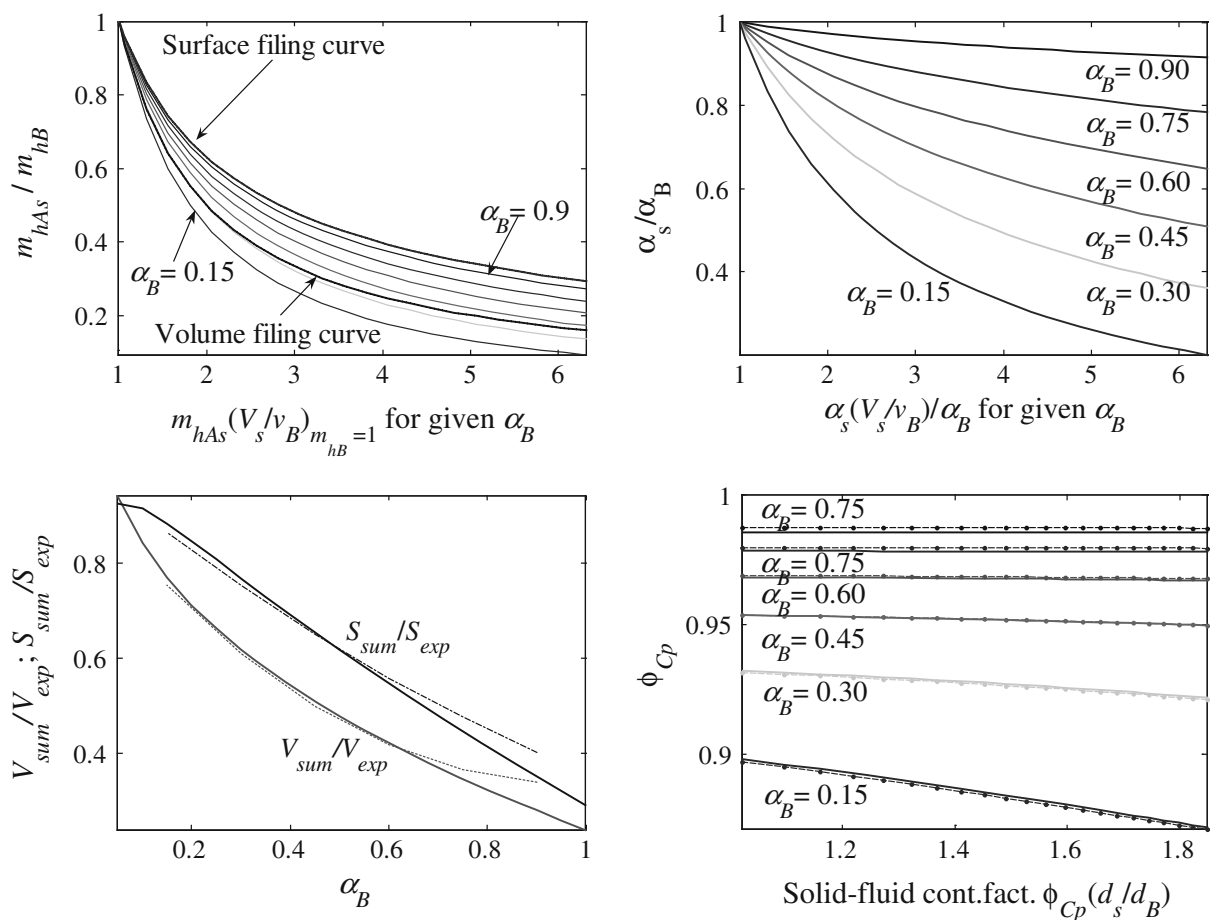

Fig. 5 Relations between LBET model parameter $\beta_{s}$ and the pore structure parameters $b$ and $\alpha_{s}$, expressed by Eqs. (21-23) (for $b=1$ the value $\beta_{s}=1$ is presumed)
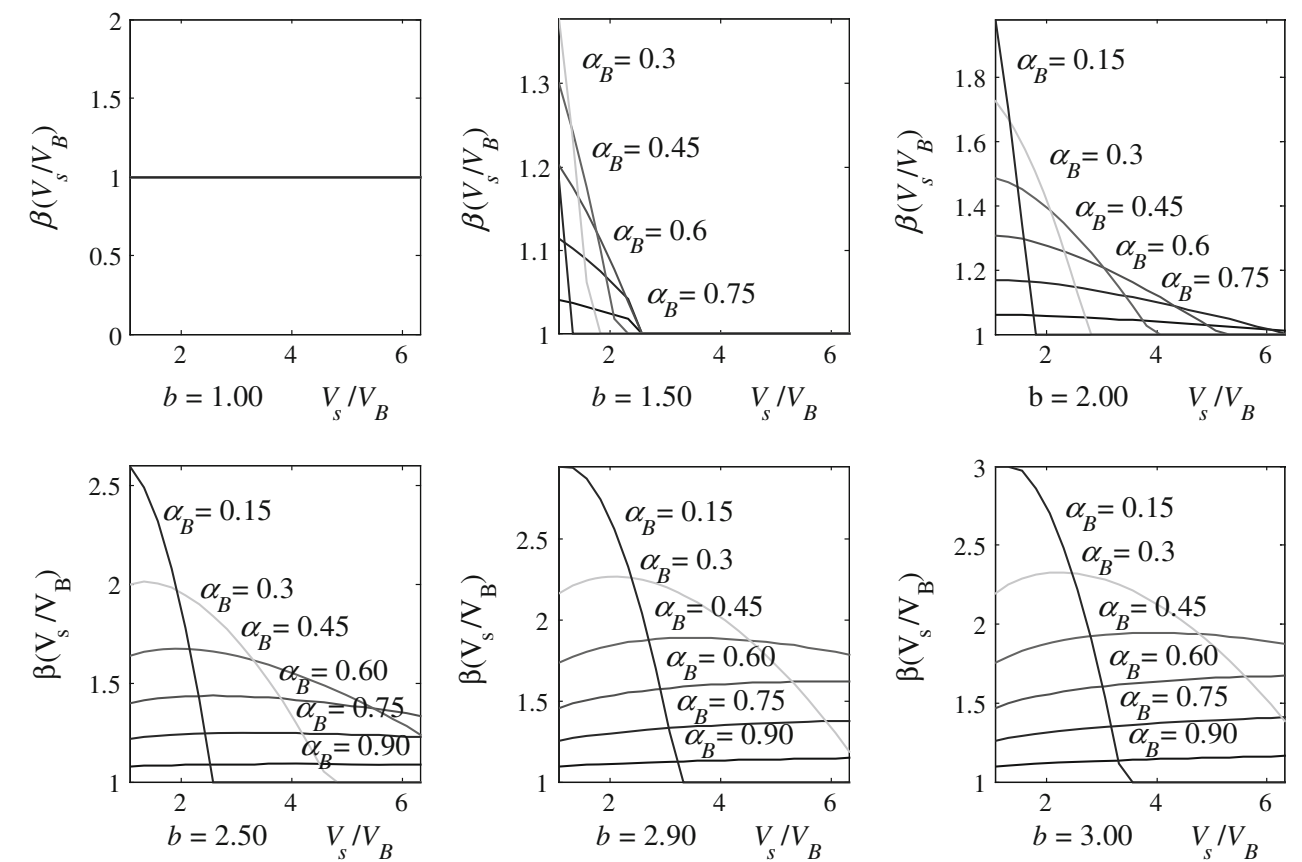

solid-fluid contact surface factor $\Phi_{C p}$. Solid lines show the factor $\Phi_{C p}$ calculated with simplified relationships as described above, and dotted-point lines - the values found with $S_{\text {exp }}$ calculated by integration of $f_{S}(h)$. We can see that the simplification errors are negligible.

Properties of the relations (21-23) between the parameter $\beta_{s}$ and the pore structure parameters $b$ and $\alpha_{s}$ are illustrated in Fig. 5. It may be seen that in narrow pores $(b<2)$ the parameter $\beta_{s}$ is strongly decreasing with 
growing $V_{s}$, but in wider ones and for $\alpha_{B}>0.5$ (that is typical in natural adsorbents) it is slightly dependent on the adsorbate molecule size and does not exceed 2.0 (which has been assumed in our earlier works).

The model parameter estimation may be noticeably improved (an uncertainty area tightened) by employing adsorption data for a number of adsorbate at the same material, employing a pore structure description invariant

Table 1 Physical parameters of adsorptives used in calculations

\begin{tabular}{lllll}
\hline The parameter & \multicolumn{4}{l}{ Adsorptive } \\
\cline { 2 - 5 } & $\mathrm{N}_{2}$ & $\mathrm{C}_{6} \mathrm{H}_{6}$ & $\mathrm{CCl}_{4}$ & $\mathrm{C}_{3} \mathrm{H}_{8}$ \\
\hline $\begin{array}{l}\text { Molar volume } V_{s}\left[\mathrm{~cm}^{3} / \mathrm{mol}\right] \\
\begin{array}{l}\text { Evaporation energy } U_{s} \\
{[\mathrm{~kJ} / \mathrm{mol}]}\end{array}\end{array}$ & 31.82 & 88.9 & 96.80 & 74.6 \\
$\begin{array}{l}\text { Temperature of the } \\
\text { adsorption process }[\mathrm{K}]\end{array}$ & 77 & 303 & 303 & 303 \\
\hline
\end{tabular}

to adsorbate molecule volume $V_{s}$. In our earlier works we have assumed three invariant parameters: a monolayer volume $V_{A}, \alpha$ and $\beta$. The relation $m_{A s}=V_{A} / V_{s}$, was proposed to evaluate $m_{A s}$ (i.e. the primary sites volume filling). Now we propose to use Eqs. (7-9, 13, 14, 16, 18, 19, 21) as the new (much more restrictive) relationships transforming the invariant parameters of random porous structure geometry $\left\{m_{0}, \alpha_{0}, b, Z_{A}, Z_{C}\right\}$ into the parameters $\left\{m_{A s}, \alpha_{s}\right.$, $\left.\beta_{s}, \xi_{A s}, \xi_{C s}\right\}$ of adsorption isotherm model (1-6) related to the adsorbate of molar volume $V_{s}$.

\section{Validation of applicability of the new identification technique}

In order to check applicability of the new approach we have used empirical adsorption isotherms of nitrogen at $77 \mathrm{~K}$, benzene, carbon tetrachloride and propane at $303 \mathrm{~K}$ on an active carbon, measured by Larionov (Larinov 1975), published by Valenzuela (Valenzuela and Myers 1989) and
Fig. 6 Results of simultaneous fitting of four isotherms on active carbon (Larinov 1975), by applying earlier (a, c subfigures) and new (b, $\mathbf{d}$ subfigures) description of random pore geometry (dotted lines show the first layer adsorption)
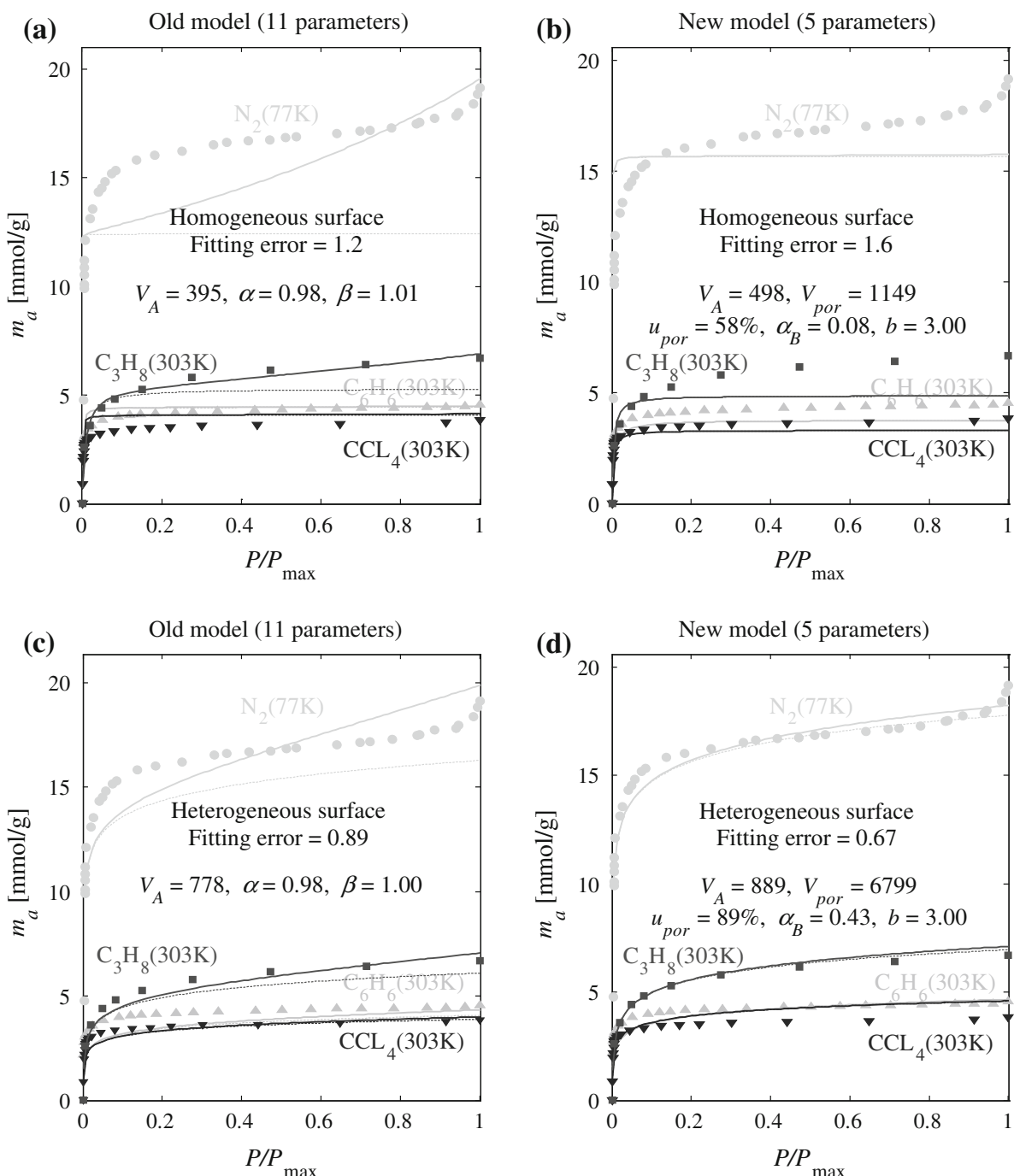
Table 2 Parameters of the LBET model obtained by simultaneous fitting of four isotherms on active carbon (Larinov 1975), by applying earlier and new description of random pore geometry (related to Fig. 6)

\begin{tabular}{|c|c|c|c|c|c|c|}
\hline \multirow[t]{2}{*}{ Figure no. } & \multicolumn{6}{|c|}{ LBET model parameters for individual adsorbates } \\
\hline & Adsorbate & $m_{h A}$ & $\alpha$ & $\beta$ & $\zeta_{A}$ & $\zeta_{C}$ \\
\hline \multirow{4}{*}{$\begin{array}{l}\text { (Fig. 6a) homogeneous } \\
\text { surface }\end{array}$} & $\mathrm{N}_{2}$ & 12.4 & \multirow[t]{4}{*}{0.98} & \multirow[t]{4}{*}{1.01} & 1.00 & 0.10 \\
\hline & $\mathrm{C}_{3} \mathrm{H}_{8}$ & 5.3 & & & 0.99 & 0.08 \\
\hline & $\mathrm{C}_{6} \mathrm{H}_{6}$ & 4.4 & & & 0.90 & 0.08 \\
\hline & $\mathrm{CCL}_{4}$ & 4.1 & & & 0.37 & 0.10 \\
\hline \multirow{4}{*}{$\begin{array}{l}\text { (Fig. 6b) homogeneous } \\
\text { surface }\end{array}$} & $\mathrm{N}_{2}$ & 15.7 & 0.04 & 1.66 & 0.42 & 0.06 \\
\hline & $\mathrm{C}_{3} \mathrm{H}_{8}$ & 4.9 & 0.01 & 1.00 & 0.40 & 0.06 \\
\hline & $\mathrm{C}_{6} \mathrm{H}_{6}$ & 3.0 & 0.01 & 1.00 & 0.39 & 0.06 \\
\hline & $\mathrm{CCL}_{4}$ & 3.3 & 0.01 & 1.00 & 0.40 & 0.06 \\
\hline \multirow{4}{*}{$\begin{array}{l}\text { (Fig. 6c) heterogeneous } \\
\text { surface }\end{array}$} & $\mathrm{N}_{2}$ & 24.5 & \multirow[t]{4}{*}{0.98} & \multirow[t]{4}{*}{1.00} & 0.52 & 0.08 \\
\hline & $\mathrm{C}_{3} \mathrm{H}_{8}$ & 10.4 & & & 0.50 & 0.12 \\
\hline & $\mathrm{C}_{6} \mathrm{H}_{6}$ & 8.8 & & & 0.85 & 0.09 \\
\hline & $\mathrm{CCL}_{4}$ & 8.0 & & & 0.51 & 0.08 \\
\hline \multirow{4}{*}{$\begin{array}{l}\text { (Fig. 6d) heterogeneous } \\
\text { surface }\end{array}$} & $\mathrm{N}_{2}$ & 27.9 & 0.35 & 1.91 & 0.49 & 0.04 \\
\hline & $\mathrm{C}_{3} \mathrm{H}_{8}$ & 12.5 & 0.25 & 1.96 & 0.48 & 0.04 \\
\hline & $\mathrm{C}_{6} \mathrm{H}_{6}$ & 10.5 & 0.23 & 1.93 & 0.48 & 0.04 \\
\hline & $\mathrm{CCL}_{4}$ & 9.7 & 0.22 & 1.90 & 0.48 & 0.04 \\
\hline
\end{tabular}

taken from (Aranovich and Donohue 1995). Data characterizing the adsorbent and physical properties of the adsorbates are gathered in Table 1.

The isotherms have been fitted simultaneously in two variants: with the formulae (1-5) (assuming surface homogeneity) and with the uniformly heterogeneous surface being assumed (Eqs. 1 and 3-6). In each variant, first earlier pore structure description was employed (assuming the same $V_{A}=m_{h A 1} \cdot V_{s 1}, \alpha$ and $\beta$ for all the isotherms) and then, the new relationships-assuming the invariant parameters $\left\{m_{0}, \alpha_{0}, b, Z_{A}, Z_{C}\right\}$-where applied. In this way the old model (involving eleven parameters to be adjusted) was compared to the new pore structure description (involving five parameters) comprising more rigorous restrictions in the fitting procedure. One may expect that these very rigorous constraints should deteriorate the fitting quality for any surface energy distribution assumed in the model, when the adsorbent structure doesn't fulfill assumptions 1-8 discussed in the Sect. 3 .

The fitting was carried out using standard optimization procedure (minimizing the fitting error standard deviation), available in MATLAB ${ }^{\circledR}$ package (MATLAB 2000) (fmincon function in MATLAB ${ }^{\circledR}$ Optimization toolbox). All calculations and plots were performed with our own software working on this platform. The results of calculations are illustrated in Fig. 6 and gathered in Table 2.

Much worse fitting quality shown in upper row subfigures than in lower ones suggests that the adsorption energy at primary sites of the studied material is not homogenous (i.e. Eq. 2 in not applicable to this adsorbent), while assuming the uniform energetic heterogeneity (Eq. 6) seems to be acceptable. As it is seen in the lower subfigures, for such a model the fitting quality is really rewarding, and even a bit better in case of the new pore structure description (right subfigure). Nevertheless, in our view the matching quality itself is of less importance here, and its worsening could be accepted as a price paid for more consistent information on the studied porous structure. Anyway the imposed constraints (7-21) of the new model were fulfilled, although the adsorption isotherm of carbon tetrachloride is overestimated.

The question is how far the compatibility of the new pore structure description allows us to predict adsorption isotherms of other small molecule adsorptives with no additional measurements. In order to answer this question we calculated firstly one isotherm using the pore structure parameters found by the simultaneous fitting of three adsorption isotherms, and secondly-two isotherms on the basis of simultaneous fitting of two isotherms. The results are shown in Fig. 7.

It is noteworthy that the calculated isotherms are almost the same as in the case of simultaneous fitting (compare lower subfigures in Fig. 7 and in Fig. 6). As the matter of fact, in each case the fitting is not perfect, but acceptable, when bearing in mind very rigorous constraints imposed by our model in Eqs. (7-19) and (21, 22).

The results presented in Fig. 7 are strong confirmation of the adequacy of the proposed pore structure description 
Fig. 7 Fitting and prediction of adsorption isotherms on the active carbon (Larinov 1975) with the new pore structure description. Left column subfigures $\mathbf{a}$ and $\mathbf{c}-$ results of simultaneous fitting of two isotherms $\left(\mathrm{N}_{2}, \mathrm{C}_{6} \mathrm{H}_{6}\right)$ and prediction of two ones (for $\mathrm{CCl}_{4}, \mathrm{C}_{3} \mathrm{H}_{8}$ ). The right subfigures $\mathbf{b}$ and $\mathbf{d}-$ results of simultaneous fitting of three isotherms $\left(\mathrm{N}_{2}, \mathrm{C}_{6} \mathrm{H}_{6}, \mathrm{CCl}_{4}\right)$ and using the pore parameters to calculate the fourth one $\left(\mathrm{C}_{3} \mathrm{H}_{8}\right)$
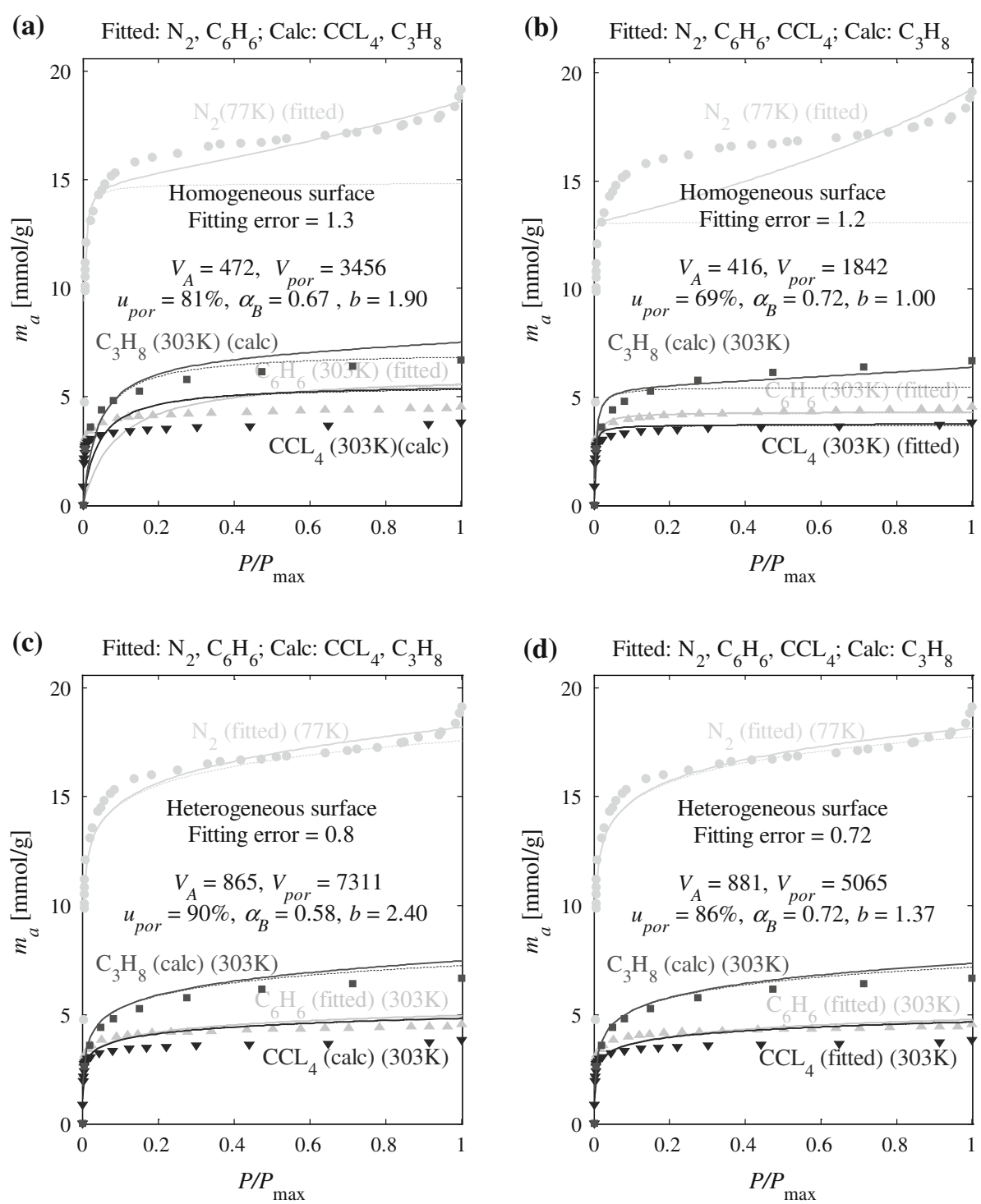

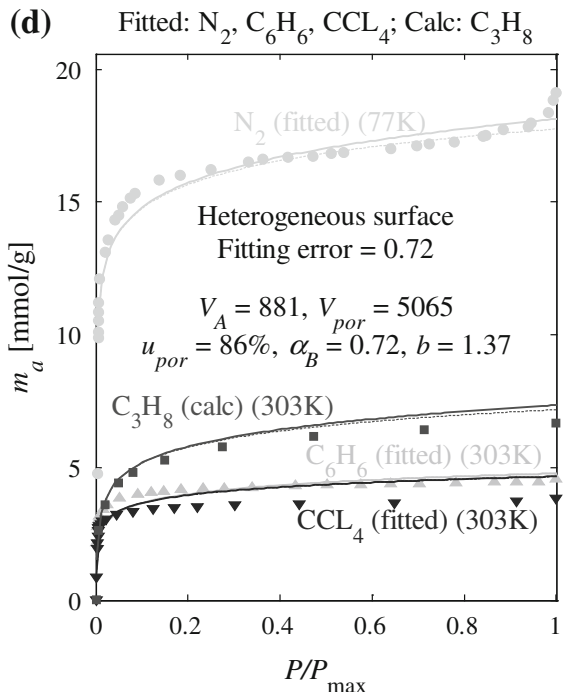

Table 3 The invariant pore structure parameters obtained in three options of calculations (assuming the uniform energetic heterogeneity of the primary adsorption sites)

\begin{tabular}{|c|c|c|c|c|c|c|}
\hline The simultaneously fitted isotherms & $V_{\text {por }} \mathrm{mm}^{3} / \mathrm{g}$ & $\alpha_{B}$ & $b$ & $Z_{\text {Amax }}$ & $Z_{C}$ & The fitting error \\
\hline $\mathrm{N}_{2}, \mathrm{C}_{6} \mathrm{H}_{6}, \mathrm{C}_{3} \mathrm{H}_{8}, \mathrm{CCl}_{4}$ & 6799 & 0.43 & 3.00 & 0.93 & 0.080 & 0.67 \\
\hline $\mathrm{N}_{2}, \mathrm{C}_{6} \mathrm{H}_{6}, \mathrm{CCl}_{4}$ & 5065 & 0.72 & 1.37 & 1.00 & 0.080 & 0.72 \\
\hline $\mathrm{N}_{2}, \mathrm{C}_{6} \mathrm{H}_{6}$ & 7311 & 0.58 & 2.40 & 1.00 & 0.096 & 0.8 \\
\hline
\end{tabular}

to examine natural adsorbents. Its main advantage is the consistency of an acquired porous structure picture, hence it can be gained by employing only two probing adsorbates (differing appropriately in molecule size). A worsening of matching quality observed in Fig. 7 (when compared to Fig. 6) seems to be fairly acceptable as a price paid for reduction of the material examination costs.
We have to notice that the parameters found in those three cases are different (see Tables 3, 4). It implies a disagreement in the picture of the pore structure, depending on isotherms employed in identification procedure, what is shown in Fig. 8. It may be seen in Fig. 8 that the differences between pore size distributions $\mathrm{g}(\mathrm{h}), G(h)$ and volume distributions $f_{v}(h)$ and $F_{v}(h)$ are fairly large (especially 
Table 4 Parameters of the LBET model obtained by simultaneous fitting of two and three isotherms (adsorbates emerged with bold letters related to Fig. 7) on the active carbon (Larinov 1975), and calculated with Eqs. (7-21) for remaining adsorbates (italic letters)

Simultaneously fitted LBET model parameters for individual isotherms (Fig. no)

\begin{tabular}{|c|c|c|c|c|c|c|}
\hline & Adsorbate & $m_{h A s}$ & $\alpha_{s}$ & $\beta_{s}$ & $\zeta_{A}$ & $\zeta_{C}$ \\
\hline \multirow{4}{*}{$\begin{array}{l}\mathrm{N}_{2}, \mathrm{C}_{6} \mathrm{H}_{6}(\text { Fig. } 7 \mathrm{a}) \\
\text { homogeneous } \\
\text { surface }\end{array}$} & $\mathbf{N}_{2}$ & 14.8 & 0.61 & 1.19 & 0.33 & 0.0 \\
\hline & $\mathrm{C}_{3} \mathrm{H}_{8}$ & 7.1 & 0.51 & 1.02 & 0.32 & 0.05 \\
\hline & $\mathrm{C}_{6} \mathrm{H}_{6}$ & 6.0 & 0.49 & 1.00 & 0.32 & 0.09 \\
\hline & $C C L_{4}$ & 5.5 & 0.48 & 1.00 & 0.32 & 0.0 \\
\hline \multirow{4}{*}{$\begin{array}{l}\mathrm{N}_{2}, \mathrm{C}_{6} \mathrm{H}_{6}, \mathrm{CCl}_{4} \\
\text { (Fig. } 7 \mathrm{~b} \text { ) } \\
\text { homogeneous } \\
\text { surface }\end{array}$} & $\mathbf{N}_{2}$ & 13.1 & 0.66 & 1.00 & 0.40 & 0.1 \\
\hline & $\mathrm{C}_{3} \mathrm{H}_{8}$ & 5.5 & 0.57 & 1.00 & 0.39 & 0.1 \\
\hline & $\mathrm{C}_{6} \mathrm{H}_{6}$ & 4.3 & 0.55 & 1.00 & 0.38 & 0.1 \\
\hline & $\mathrm{CCL}_{4}$ & 3.7 & 0.55 & 1.00 & 0.39 & 0.1 \\
\hline \multirow{4}{*}{$\begin{array}{l}\mathrm{N}_{2}, \mathrm{C}_{6} \mathrm{H}_{6} \text { (Fig. 7c) } \\
\text { heterogeneous } \\
\text { surface-accepted }\end{array}$} & $\mathbf{N}_{2}$ & 27.2 & 0.51 & 1.43 & 0.50 & 0.0 \\
\hline & $\mathrm{C}_{3} \mathrm{H}_{8}$ & 12.8 & 0.41 & 1.35 & 0.49 & 0.0 \\
\hline & $\mathrm{C}_{6} \mathrm{H}_{6}$ & 10.8 & 0.38 & 1.30 & 0.49 & 0.0 \\
\hline & $\mathrm{CCL}_{4}$ & 10.0 & 0.37 & 1.27 & 0.50 & 0.0 \\
\hline \multirow{4}{*}{$\begin{array}{l}\mathrm{N}_{2}, \mathrm{C}_{6} \mathrm{H}_{6}, \mathrm{CCl}_{4} \\
\text { (Fig. 7d) } \\
\text { heterogeneous } \\
\text { surface-accepted }\end{array}$} & $\mathbf{N}_{2}$ & 27.7 & 0.66 & 1.00 & 0.50 & 0.0 \\
\hline & $\mathrm{C}_{3} \mathrm{H}_{8}$ & 12.9 & 0.58 & 1.00 & 0.48 & 0.0 \\
\hline & $\mathbf{C}_{6} \mathbf{H}_{6}$ & 10.7 & 0.56 & 1.00 & 0.48 & 0.04 \\
\hline & $\mathrm{CCL}_{4}$ & 9.8 & 0.55 & 1.00 & 0.49 & 0.04 \\
\hline
\end{tabular}

for larger $h$ ), while the surface distributions $F_{s}(h)$ are much closer. It means that the total number of pores $G(\infty)$ and total pore volume $V_{\text {sum }}=F_{v}(\infty)$, as well as the pore size, volume and surface distribution in a medium pore size range, do not affect significantly adsorption properties of the studied material (quite different curves yield similar isotherms). On the other hand, almost identical curves
$G(h)$ for $h<1 \mathrm{~nm}$ reveal the crucial effect of the number of very small pores on the material adsorptivity. Also the pore surface distribution seems to be of importance, as the curves $F_{S}(h)$, especially $S_{\text {sum }}=F_{S}(\infty)$ are rather close. Thus, the curves presented in Fig. 8 may be viewed as a picture of uncertainty of the structure parameters found on the basis of adsorption measurements.

\section{Conclusions}

The numerical tools presented in this paper are significant development of works lead in the Authors team, focused on mathematical modeling of adsorption process and aimed at identification of porous structures properties. The proposed consistent description of random porous structures related to the adsorption model (1-6) makes possible to get quantitative information on the pores shape, size and volume by simultaneous fitting of a number of isotherms measured at the studied material.

Obtained acceptable quality of the fitting allows one to take that the structure of the material fulfills assumptions of the proposed model. Hence it enables one to predict isotherms of other adsorptives, on the basis of their physical parameters and invariant parameters of the pore structure found before. This predictivity is the main advantage of the presented approach, as it makes possible to reduce the costs of natural adsorbents examination.

The newly elaborated tool may be also employed as a support in the natural adsorbent production technology, by analyzing the influence of pore structures on adsorption capacity by simulation experiments.
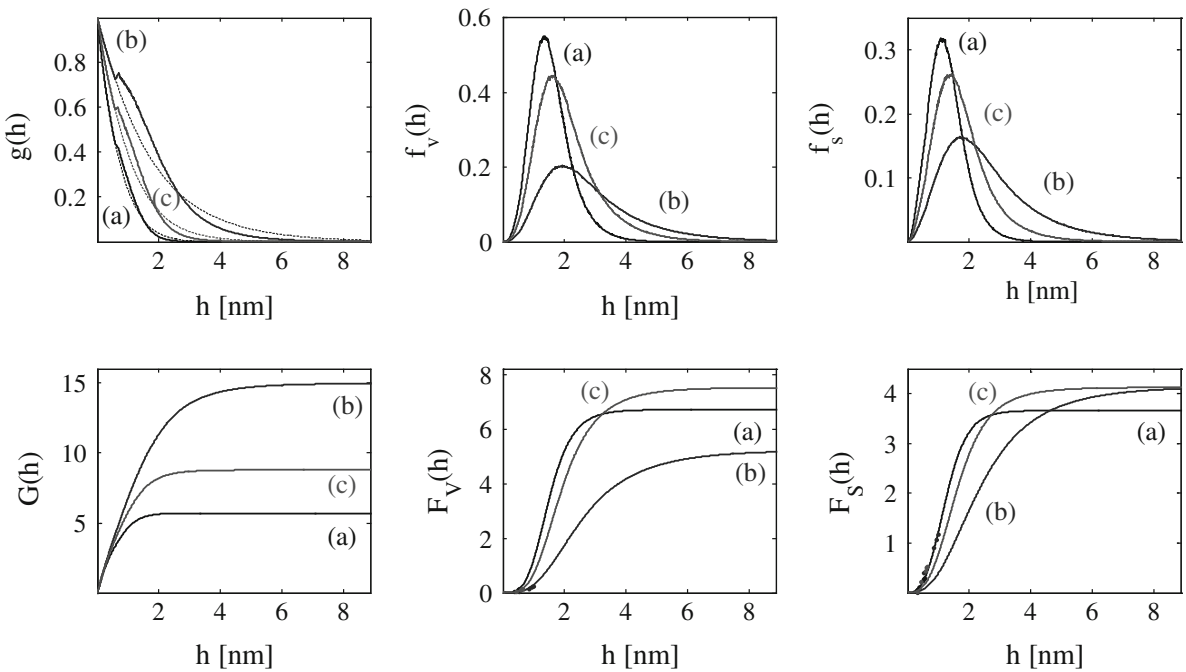

Fig. 8 Pore size, volume and surface distributions at the active carbon (Larinov 1975) found by simultaneous fitting of four $(a)$, three $(b)$ and two (c) isotherms-the pore structure parameters are: a $u_{p}=89 \% \alpha_{B}=0.43, b=3$; b $u_{p}=86 \% \alpha_{B}=0.72, b=1.37$; c $u_{p}=90 \% \alpha_{B}=0.58$, $b=2.4$ 
Acknowledgments The research is led within the AGH University of Science and Technology in Krakow Grant No. 11.11.210.213.Open Access This article is distributed under the terms of the Creative Commons Attribution License which permits any use, distribution, and reproduction in any medium, provided the original author(s) and the source are credited.

\section{References}

Aranovich, G.L., Donohue, M.D.: Adsorption isotherms for microporous adsorbents. Carbon 33, 1369-1375 (1995)

Clarkson, C.R., Bustin, R.M., Levy, J.H.: Application of the mono/ multilayer and adsorption potential theories to coal methane adsorption isotherms at elevated temperature and pressure. Carbon 35, 1689-1705 (1997)

Duda, J.T., Milewska-Duda, J.: Mathematical description of constrained BET-like adsorption and its approximation with LgBET and LcBET formulas. Langmuir J. AChS 18, 7503-7514 (2002)

Duda, J.T., Milewska-Duda, J.: Modeling of surface heterogeneity of microporous adsorbents with LBET approach. Langmuir J. AChS 21, 7243-7256 (2005)
Duda, J.T., Kwiatkowski, M., Milewska-Duda, J.: Computer modeling and analysis of heterogeneous structures of microporous carbonaceous materials. J. Mol. Model. 11(4-5), 416-430 (2005)

Duda, J.T., Jagiełł, L., Jagiełło, J., Milewska-Duda, J.: Complementary study of microporous adsorbents with DFT and LBET. Appl. Surf. Sci. 253, 5616-5621 (2007)

Jagiello, J., Ansón, A., Martínez, M.T.: DFT-based prediction of highpressure $\mathrm{H}_{2}$ adsorption on porous carbons at ambient temperatures from low-pressure adsorption at $77 \mathrm{~K}$. J. Phys. Chem. B 110, 4531-4534 (2006)

Jaroniec, M., Madey, R.: Physical adsorption on heterogenous surfaces. Elsevier, Amsterdam (1988)

Kats, B.M., Kutarov, V.V.: A modified BET equation for polylayer adsorption. Adsorpt. Sci. Technol. 16(4), 257-262 (1998)

Larinov, O.G.: Adsorption of individual vapors and solutions of nonelectrolytes. Thesis, Institute of Physical Chemistry, Moscow (1975)

MATLAB ${ }^{\circledR}$ The Language of Technical Computing, v.6.0.0.88Reference Manual (2000)

Tarazona, P., Marini Bettolo Marconi, U., Evans, R.: Phase-equilibria of fluid interfaces and confined fluids - nonlocal versus local density functionals. Mol. Phys. 60(3), 573-595 (1987)

Valenzuela, D.P., Myers, A.L.: In Adsorption Equilibrium. Prentice Hall, Englewood Cliffs (1989) 